\title{
Phonon renormalization effects in photoexcited quantum wires
}

\author{
K. Güven and B. Tanatar \\ Department of Physics, Bilkent University, Bilkent, 06533 Ankara, Turkey
}

(Received 5 August 1994)

\begin{abstract}
We study the effects of screening on polaronic corrections to the effective band edge in a quasione-dimensional GaAs quantum wire. We find that the screening effects and finite well width considerably reduce the polaron energy and oppose the polaronic band-gap renormalization. We calculate the polaronic effective mass as a function of the carrier density and temperature. Effects of the vertex corrections to the conduction- and valence-band edges are also discussed.
\end{abstract}

\section{INTRODUCTION}

Formation of a dense electron-hole plasma in a semiconductor under intense laser excitation is a well-known phenomenon. Because of the exchange-correlation effects and the screening of the Coulomb interaction, many single-particle properties in the system are renormalized, of which the most dramatic one is the band-gap renormalization (also known as the band-gap shrinkage) as a function of the plasma density. This is important to determine the emission wavelength of coherent emitters as being used in semiconductors. ${ }^{1}$ Since a substantial carrier population may be induced by optical excitation, the renormalized band gap can affect the excitation process in turn and lead to optical nonlinearities. On the other hand, the coupling between the charge carriers and LO phonons in these systems also affects the band-gap energy and carrier effective mass. The gap between the valence and conduction bands is renormalized by the emission and absorption of LO phonons. In this paper we investigate the density and temperature dependence of the band-gap renormalization (BGR) in quasione-dimensional photoexcited semiconductors due to the phonon effects within the perturbation theoretical approach.

Under high optical excitation the band gap for twodimensional (2D) and three-dimensional (3D) systems is found to decrease with increasing plasma density due to exchange-correlation effects. The observed band gaps are typically renormalized by $\sim 20 \mathrm{meV}$ within the range of plasma densities of interest which arise solely from the conduction-band electrons and valence-band holes. In the quasi-one-dimentional structures based on the confinement of electrons and holes, the electron-hole plasma is quantized in two transverse directions, thus the charge carriers essentially move only in the longitudinal direction. Recent progress in the fabrication techniques such as molecular-beam epitaxy and lithographic deposition have made possible the realization of such quasione-dimensional systems. ${ }^{2,3}$ Band-gap renormalization as well as various optical properties of the electron-hole systems have been studied for bulk (3D) and quantumwell two-dimensional (2D) semiconductors, ${ }^{4,5}$ providing generally good agreement with the corresponding measurements. ${ }^{6}$

Two processes contribute to the band gap renormalization. The interaction of the electron-hole pair with the thermal phonons causes a decrease of the band gap with increasing temperature, while the exchangecorrelation effects cause a decrease in the band gap with increasing plasma density. The band gap between the valence and conduction bands in GaAs is about $\sim 1.5 \mathrm{eV}$. The exchange-correlation-induced BGR in quantum wires may be as large as $\sim 25 \mathrm{meV}$ according to recent measurements ${ }^{7}$ and calculations. ${ }^{8,9}$ Our calculation of the renormalization due to LO-phonon coupling is of the same order. The polaronic renormalization is always present and should be subtracted from the total BGR. In a more complete theory, ${ }^{10,11}$ of the band gap renormalization in photoexcited semiconductor structures, the effective interaction $V(q, E)$, which consists of the bare Coulomb and LO-phononmediated carrier-carrier interaction including the dynamical screening, should be used.

In this study our aim is to calculate the BGR due to polaron effects using a statically screened approximation which is based on the random-phase approximation (RPA). We employ the temperature-dependent, static, RPA dielectric function and address also the question of validity of using the plasmon-pole approximation to it. We investigate the temperature and electron-hole plasma density dependence of the BGR at various quantum-well widths. Electron-hole-LO-phonon coupling in quasi-onedimensional systems depends on the well width, freecarrier density, and temperature, which we discuss in detail. Since the screening function $\varepsilon(q)$ determines these quantities, we investigate different models and attempt to include the vertex corrections in an approximate way. Although it has been shown that in semiconductors of reduced dimensionality, confined and interface phonon modes have substantial effects, ${ }^{12}$ we ignore them here and consider only the coupling of electronhole plasma to bulk phonons. Phonon renormalization effects in two-dimensional (2D) quantum wells were studied by Das Sarma and Stopa, and Xiaoguang et al. ${ }^{13}$ The screening of the electron-phonon interaction in quasi-onedimentional structures within a variational approach was considered by Hai et al. ${ }^{14}$ 
The rest of this paper is organized as follows. In the next section we give a brief outline of the model of quasi-one-dimensional system we use, the electronhole-phonon self-energy at the band edges within the static screening approximation, and mass renormalization. In Sec. III we present our results for the LOphonon-induced band gap renormalization in quasi-onedimensional electron-hole plasmas and the changes in the effective electron and hole masses. Finally, we conclude with a brief summary of our main results.

\section{THEORY}

For the two-component quasi-one-dimensional system consisting of electrons and holes, we consider a square well of width $a$ with infinite barriers. It may be built from a quasi-two-dimensional quantum well (grown in the $z$ direction) by introducing an additional lateral confinement. We assume that the effective mass approximation holds and for GaAs take $m_{e}=0.067 m$ and $m_{h}=0.4 m$, where $m$ is the bare (free) electron mass. The effective Coulomb interaction between the charge carriers in their lowest subband is given by ${ }^{8}$ the average over the subband wave functions

$$
\begin{aligned}
V(q)= & \frac{2 e^{2}}{\epsilon_{0}} \int_{0}^{1} d x K_{0}(q a x)[2-(1-x) \cos (2 \pi x) \\
& \left.+\frac{3}{2 \pi} \sin (2 \pi x)\right],
\end{aligned}
$$

in which $K_{0}(x)$ is the zeroth-order modified Bessel function of the second kind and $\epsilon_{0}$ is the lattice dielectric constant. We express the above equation as $V(q)=$ $2 e^{2} F(q) / \epsilon_{0}$ for subsequent usage. Optical excitation creates an electron-hole plasma, and due to the presence of this two-component plasma, assumed to be in equilibrium, the bare Coulomb interaction is screened. The equilibrium assumption is justified since the laser pulse durations are typically much longer than the relaxation times of the semiconductor structures under study.

The self-energy (real part) due to electron-hole-LOphonon interaction for a quasi-one-dimensional system, within the static screening approximation, is given by ${ }^{15}$

$$
\begin{aligned}
\operatorname{Re} \Sigma_{e, h}(k, E)= & \frac{2 \alpha_{e, h}}{\pi} \frac{\omega_{\mathrm{LO}}^{2}}{\sqrt{2 m_{e, h} \omega_{\mathrm{LO}}}} \int_{0}^{\infty} d q \frac{F(q)}{[\varepsilon(q, 0)]^{2}} \\
& \times\left[\frac{n_{0}+f_{e, h}(k-q)}{E+\omega_{\mathrm{LO}}-\epsilon_{e, h}(k-q)}\right. \\
& \left.+\frac{n_{0}+1-f_{e, h}(k-q)}{E-\omega_{\mathrm{LO}}-\epsilon_{e, h}(k-q)}\right]
\end{aligned}
$$

where $\alpha_{e, h}$ is the Fröhlich coupling constant for electrons or holes defined as

$$
\alpha_{e, h}=\frac{1}{2}\left(\frac{1}{\epsilon_{\infty}}-\frac{1}{\epsilon_{0}}\right) \frac{e^{2}}{\omega_{\mathrm{LO}}} \sqrt{2 m_{e, h} \omega_{\mathrm{LO}}} .
$$

In the above equations, $\epsilon_{\infty}$ is the optical dielectric con- stant, $\omega_{\mathrm{LO}}=36.5 \mathrm{meV}$ is the bulk LO-phonon energy in GaAs, and $n_{0}$ and $f_{e, h}(k)$ are the Bose (phonon) and Fermi occupancy factors, respectively.

The main assumptions in writing the self-energy due to electron-hole-phonon coupling in the above form are as follows. First, the electron-phonon coupling is expressed in terms of the Fröhlich Hamiltonian. The LO phonons are treated without any dispersion. The bare electron propagator $G_{0}(k, E)$ is used rather than solving the Dyson's equation self-consistently. Along with the Bose distribution functions $n_{0}$, we have also retained the Fermi surface effects through the Fermi distribution functions $f_{e, h}(k)$. We will show later that when the Fermi energy $E_{F} \ll \omega_{\mathrm{LO}}$, the Fermi occupancy effects will not be important, as in the case of two-dimensional (2D) systems. ${ }^{13}$ Finally, we assume the quantum size limit for which both the electrons and holes remain in their respective lowest subbands of the quantum well.

We work in the static screening approximation, and employ the static RPA for the dielectric function

$$
\begin{aligned}
\varepsilon(q, E & =0, T) \\
& =1-V(q)\left[\Pi_{e}(q, E=0, T)+\Pi_{h}(q, E=0, T)\right],
\end{aligned}
$$

where $V(q)$ is the Coulomb interaction between the charged particles and $\Pi_{e, h}(q, E=0, T)$ are the finitetemperature static polarizabilities for electrons and holes. The form we use for $\varepsilon(q, 0)$ is appropriate for a photoexcited intrinsic semiconductor since screening by electrons and by holes are treated on an equal footing. In the case of doped $n$ - and $p$-type semiconductors, screening by electrons and by holes should be considered separately. We calculate the finite-temperature polarizabilities using the Maldague ${ }^{16}$ approach starting from the zero-temperature quasi-one-dimensional polarizability of an electron gas

$$
\begin{aligned}
\Pi_{e, h}(q, E & =0, T) \\
& =\frac{m_{e, h}}{\pi q} \int_{0}^{\infty} d t \ln \left|\frac{y+\sqrt{t}}{y-\sqrt{t}}\right| \frac{1}{\cosh ^{2}(x / 2-t)},
\end{aligned}
$$

where $x=\mu_{e, h} / T$ and $y=q / 4 \sqrt{m_{e, h} T}$ (we take Planck's constant $\hbar$ and the Boltzmann constant $k_{B}$ to be equal to 1). Here $\mu_{e, h}$ are the chemical potentials for each species at finite temperature. In various applications, the dielectric function $\varepsilon(q)$ was further simplified by the plasmon-pole approximation. Here we use the full static RPA at finite temperature without resorting to any approximations and discuss in Sec. III the validity of the plasmon-pole approximation.

We make the usual assumption of parabolic bands, taking the electron and hole single-particle energies to be $\epsilon_{e, h}(k)=k^{2} / 2 m_{e, h}$. This should be justified for the GaAs example we consider in this work, but for certain other semiconductors such as InSb, nonparabolicity effects would require higher-order corrections. In addition, we evaluate the electron-phonon self-energies on the mass shell $\left(E=k^{2} / 2 m_{e, h}\right)$ to obtain the polaronic corrections at the band edge $\left[\operatorname{Re} \Sigma_{e, h}(0,0)\right]$ 


$$
\begin{aligned}
E_{p}= & -\frac{2 \alpha_{e, h}}{\pi} \frac{\omega_{\mathrm{LO}}^{2}}{\sqrt{2 m_{e, h} \omega_{\mathrm{LO}}}} \int_{0}^{\infty} d q \frac{F(q)}{[\varepsilon(q)]^{2}} \\
& \times \frac{\left(2 n_{0}+1\right)\left(q^{2} / 2 m_{e, h}\right)+\left[2 f_{e, h}(q)-1\right] \omega_{\mathrm{LO}}}{\left(q^{2} / 2 m_{e, h}\right)^{2}-\omega_{\mathrm{LO}}^{2}} .
\end{aligned}
$$

The limit $\varepsilon \rightarrow 1$ (no screening) renders the above polaronic energy independent of the carrier density, if we further neglect the Fermi occupancy factors. Unlike the case of two-dimensional (2D) systems, a closed form expression for $E_{p}$ in the no-screening limit is not possible.

The definition of the polaron effective mass (in the long-wavelength limit) is given by

$$
\frac{1}{m_{e, h}^{*}}=\frac{1}{m_{e, h}}+\lim _{k \rightarrow 0} \frac{1}{k} \frac{\partial}{\partial k} \operatorname{Re} \Sigma_{e, h}\left(k, k^{2} / 2 m\right) .
$$

For low temperatures $(T \leq 50 \mathrm{~K})$, we neglect the phonon occupancy, take $n_{0} \rightarrow 0$, expand the remaining integrand in powers of $k$, take the derivative, and let $k \rightarrow 0$, thus obtaining

$$
\begin{aligned}
\frac{1}{m_{e, h}^{*}}= & \frac{1}{m_{e, h}}-\frac{\alpha_{e, h}}{m_{e, h}}\left[\frac{2}{\pi} \frac{\omega_{\mathrm{LO}}^{2}}{\sqrt{2 m_{e, h} \omega_{\mathrm{LO}}}} \frac{2}{m_{e, h}}\right. \\
& \left.\times \int_{0}^{\infty} d q \frac{F(q)}{[\varepsilon(q)]^{2}} \frac{q^{2}}{\left[\omega_{\mathrm{LO}}+q^{2} / 2 m_{e, h}\right]^{3}}\right] .
\end{aligned}
$$

The above expression yields in the weak coupling limit $\left(\alpha_{e, h} \rightarrow 0\right) m_{e, h}^{*} \approx m_{e, h}(1+\alpha C)$, where $C$ is given by the expression inside the large square brackets in Eq. (8).

\section{RESULTS AND DISCUSSION}

We begin by presenting our results for the band-edge polaronic corrections at low temperatures. Since the dielectric function $\varepsilon(q)$ of a quasi-one-dimensional system diverges at $2 k_{F}$ and $T=0$, we choose a small but finite temperature to work with. Figures 1(a) and 1(b) show the electron and hole polaron energies, respectively, as a function of the carrier density $N$ for various well widths at $T=5 \mathrm{~K}$. The solid curves in both figures, from top to bottom, indicate widths of $a=500,250$, and $100 \AA$. In order to see the influence of the Fermi occupancy factors we also plot by dashed lines $E_{p}$ calculated without $f_{e, h}$. In the density range of interest, they are negligibly small, except close to $N \sim 10^{6} \mathrm{~cm}^{-1}$ both for electrons and holes. Since $E_{F} \sim k_{F}^{2} \sim N^{2}$, it turns out that the condition $E_{F} \ll \omega_{\text {LO }}$ breaks down for $N>10^{6} \mathrm{~cm}^{-1}$. Also shown in these figures by horizontal dotted lines are the unscreened energies. They are calculated using Eq. (6) with $\varepsilon(q) \rightarrow 1, n_{0} \rightarrow 0$, and $f_{e, h} \rightarrow 0$. The no-screening limit depends only on the well width and typical numbers are $E_{p}=-3.879,-2.403$, and $-1.575 \mathrm{meV}$ for well widths of $a=100,250$, and $500 \AA$, respectively, for the case of electrons. The corresponding values for holes are $E_{p}=-6.631,-3.773$, and $-2.340 \mathrm{meV}$.

In Figs. 2(a) and 2(b), we show the polaronic correction to the band gap as a function of plasma density at $a=100 \AA$. The solid lines indicate, from top to bottom,
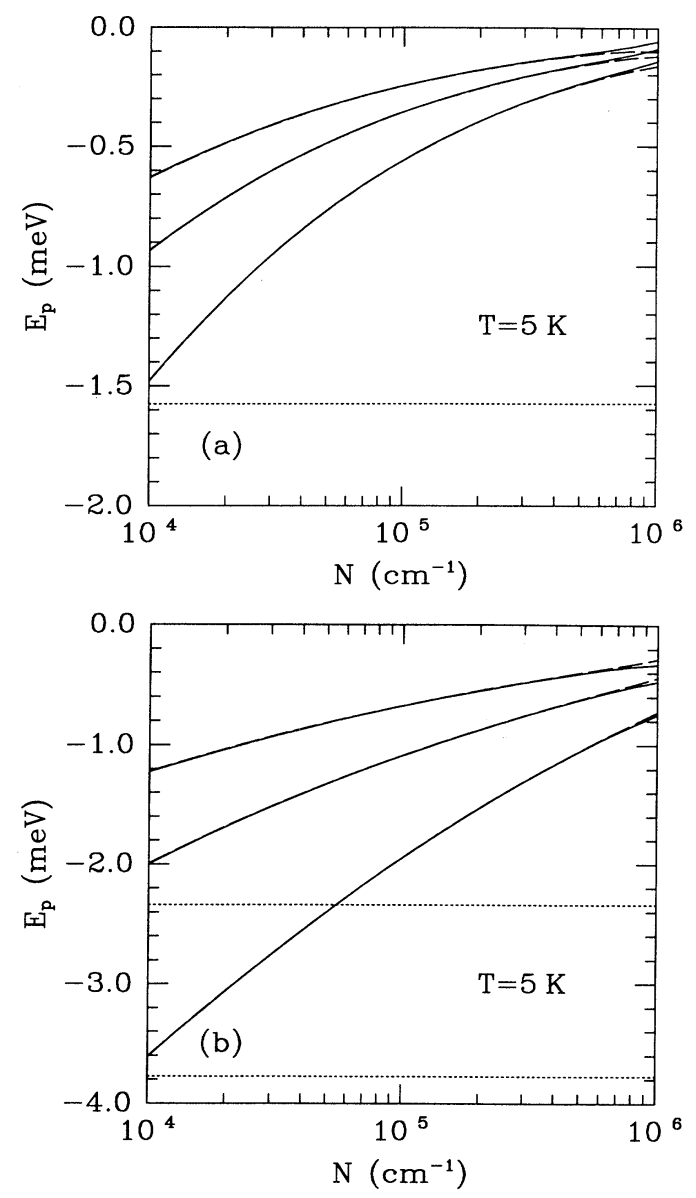

FIG. 1. (a) Polaron correction to the conduction-band edge as a function of the carrier density $N$ at $T=5 \mathrm{~K}$. Solid (dashed) lines from top to bottom are for well widths $a=500$, 250, and $100 \AA$, with (without) Fermi surface effects. The corresponding dotted lines indicate the unscreened limits. (b) Same for the valence-band edge.

$T=5,100$, and $300 \mathrm{~K}$. We note that as the temperature increases, $E_{p}$ also increases in magnitude. As a general trend, the phonon renormalization decreases for higher values of the carrier density, while its rate is temperature dependent. The dashed curves in Fig. 2 gives the BGR calculated within the plasmon-pole approximation to the dielectric function using the same parameters. In the plasmon-pole approximation the static dielectric function is expressed as $^{9}$

$$
\varepsilon(q)=1+\sum_{i=e, h} \frac{\omega_{p, i}^{2}}{\left(N q^{2} / m_{i} \kappa_{i}\right)+\left(q^{2} / 2 m_{i}\right)^{2}},
$$

where the plasmon frequency for the quasi-onedimensional ${ }^{17}$ system is $\omega_{p, i}^{2}=\left(N / m_{i}\right) V(q)$ (in the long-wavelength limit) and the screening parameter is $\kappa_{i}=\partial N / \partial \mu_{i}$. The plasmon-pole approximation consists of ignoring the weight of single-particle excitations and assuming that all the weight of the dynamic susceptibility $\Pi_{0}(q, \omega)$ is at an effective plasmon energy $\omega_{p}$. It cor- 

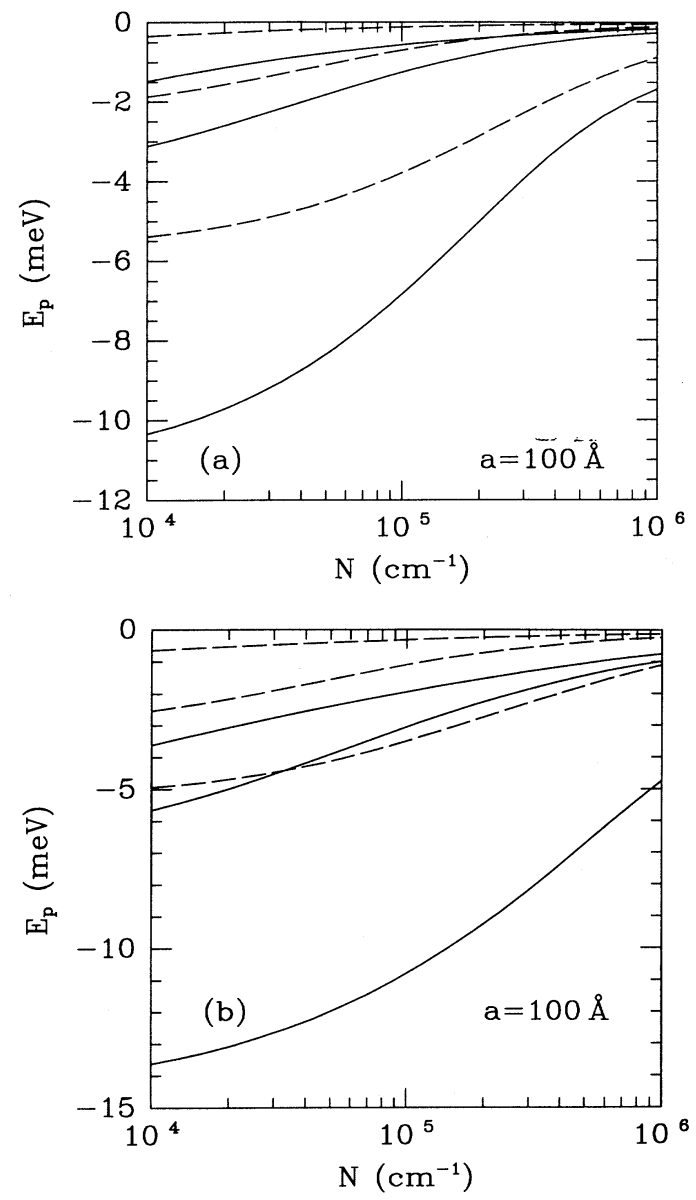

FIG. 2. (a) Polaron correction to the conduction-band edge as a function of the carrier density $N$ for a quantum-well wire of width $a=100 \AA$. The solid lines from top to bottom indicate $T=0,100$, and $300 \mathrm{~K}$ calculated with full RPA, whereas the dashed lines are with the plasmon-pole approximation. (b) Same for the valence-band edge.

rectly describes the static and long-wavelength limits of the full RPA expression. We note that the temperaturedependent plasmon-pole approximation to the dielectric function yields considerably different results from the RPA. Das Sarma et al. ${ }^{10}$ have found significant deviations of the plasmon-pole approximation from the full RPA results in two-dimensional (2D) quantum wells. Our calculations suggest increasing discrepancies between the full RPA and plasmon-pole approximation as $T$ increases. The temperature dependence in the plasmon pole approximation mainly enter through the screening parameter $\kappa$ and it is conceivable that differences originate from somewhat different temperature dependences.

Having established the insignificance of the Fermi occupancy factors in the polaronic correction to the band gap renormalization in the density range of interest $\left(10^{4}<N<10^{6} \mathrm{~cm}^{-1}\right)$, we now turn to the temperature dependence of $E_{p}$. Self-energy increases in magnitude as the carrier temperature is raised. At low temperatures, $E_{p}$ is due mainly to virtual phonons, since $n_{0}$ (the av- erage number of real phonons in the system) becomes vanishingly small as $T \rightarrow 0$. At higher temperatures, the average phonon number increases and emission and absorption of phonons contribute to $E_{p}$ through the factors $n_{0}$ and $n_{0}+1$ in Eq. (6).

Figure 3 shows the effects of finite temperature on the polaronic energy. In Fig. 3(a) we display the conductionband correction as a function of $T$, for various carrier densities, in a quantum wire of well width $a=200 \AA$. Solid lines from top to bottom are for $N=10^{4}, 10^{5}$, and $10^{6} \mathrm{~cm}^{-1}$, respectively. In Fig. $3(\mathrm{~b})$, the same quantity is plotted for the valence band. The dashed lines in Fig. 3 are calculated without the phonon occupancy factors $n_{0}$, but we retain the dielectric function $\varepsilon(q)$. The difference between the dashed line and the corresponding solid line is a measure of the thermal phonon effects, which seem to be rather important for $T>100 \mathrm{~K}$. The dotted lines in Figs. 3(a) and 3(b), are calculated by setting $\varepsilon(q)=1$ while keeping the phonon occupancy factors. In the no-
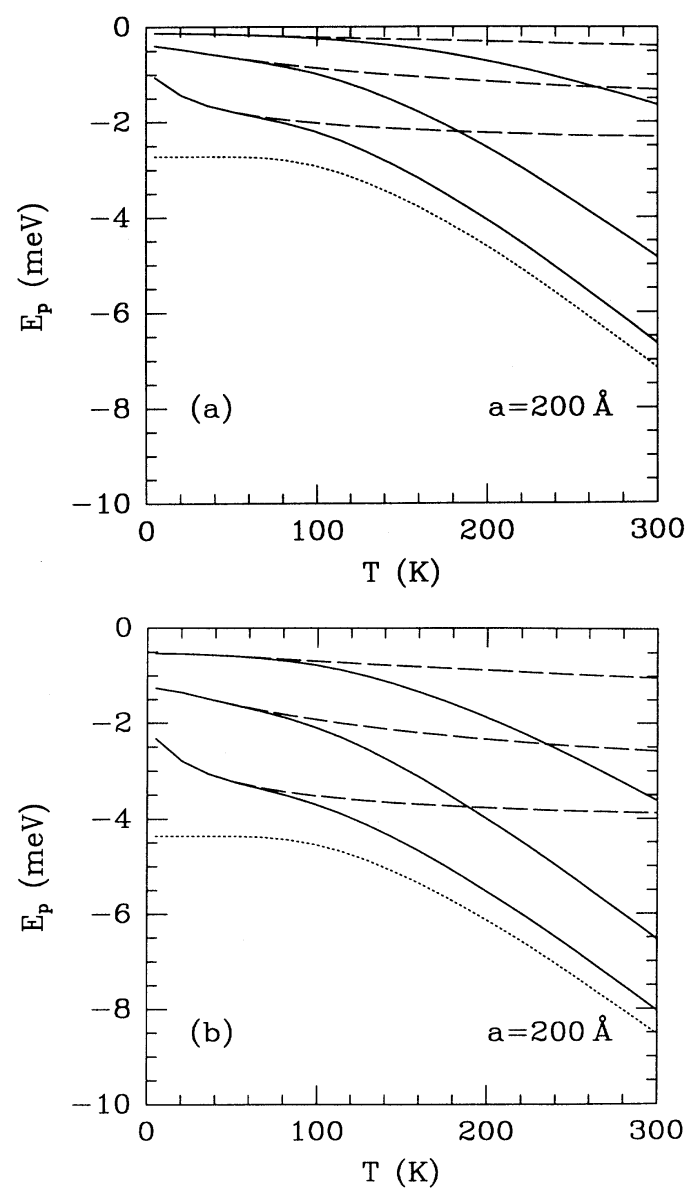

FIG. 3. (a) Temperature dependence of the polaron correction to the conduction-band edge for a quantum-well wire of width $a=200 \AA$. The solid lines from top to bottom indicate $N=10^{6}, 10^{5}$, and $10^{4} \mathrm{~cm}^{-1}$. The dashed lines show the effects of thermal phonons $\left(n_{0}=0\right)$. The dotted line is calculated in the no-screening limit. (b) Same for the valence-band edge. 
screening limit this quantity is independent of the density.

The foregoing results for the polaronic corrections at the conduction- and valence-band edges imply a total of $\sim 10 \mathrm{meV}$ renormalization in the density range $10^{4}<$ $N<10^{6} \mathrm{~cm}^{-1}$, which is comparable to the exchangecorrelation corrections. ${ }^{7-9}$ The phonon renormalization effects become negligible for densities $N>10^{6} \mathrm{~cm}^{-1} \mathrm{ir}-$ respective of the quantum wire well width. We observe these effects also for a quantum wire of width $a=500 \AA$ in Fig. 4. The solid lines in Fig. 4 are calculated with the RPA dielectric function, whereas in the dashed lines vertex corrections are included.

We now discuss the effects of local-field correction to the BGR due to polaronic effects in quantum wires. Writing the dielectric function as $\varepsilon(q)=1-V(q) \Pi(q) \gamma(q)$, where $\gamma(q)=1+V(q) G(q) \Pi(q)$ is the vertex function in which $G(q)$ is the static local-field factor and $\Pi(q)$ is the static Lindhard polarizability, we account for the vertex corrections to $\Pi(q)$ in the mean-field sense. We use the equivalent of Hubbard approximation for $G(q)$ in one
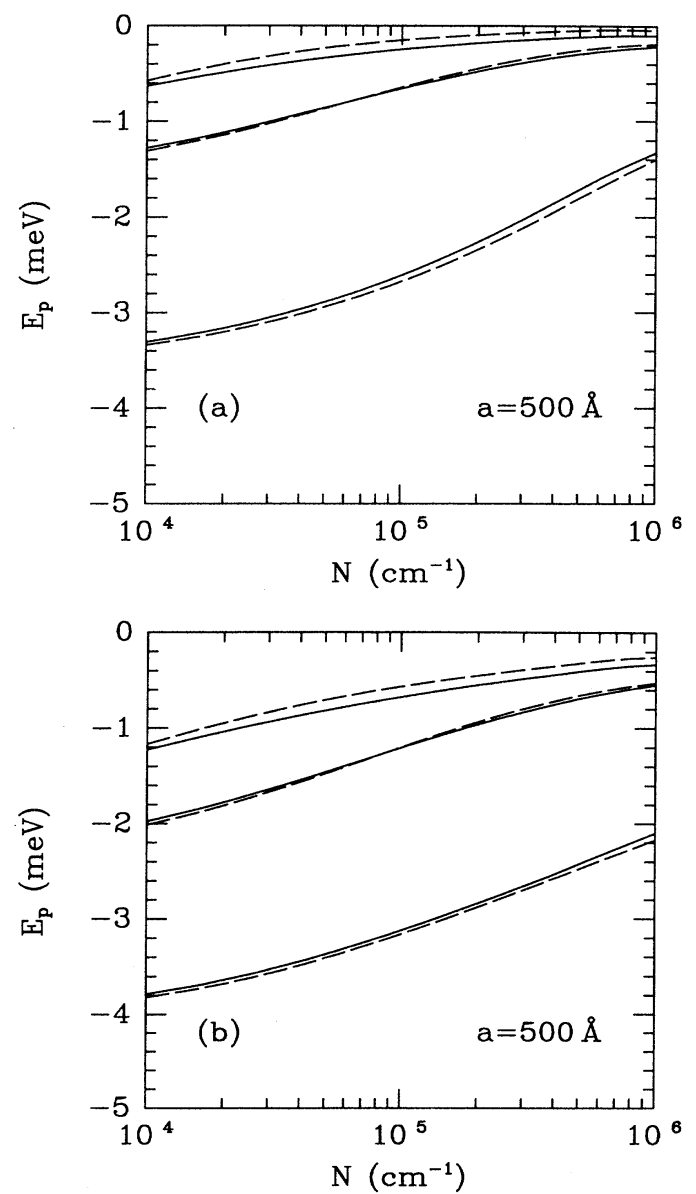

FIG. 4. (a) Polaron correction to the conduction-band edge as a function of the carrier density $N$ for a quantum-well wire of width $a=500 \AA$. The solid lines from top to bottom indicate $T=0,100$, and $300 \mathrm{~K}$. The dashed lines are calculated with a dielectric function which includes the vertex corrections. (b) Same for the valence-band edge. dimension ${ }^{18}$

$$
G(q) \approx \frac{1}{2} \frac{V\left(\sqrt{q^{2}+k_{F}^{2}}\right)}{V(q)} .
$$

The physical nature of the Hubbard approximation is such that it takes exchange into account and corresponds to using the Pauli hole in the calculation of the localfield correction between the particles of the same kind. Coulomb correlations are omitted. In this simple form, the static local-field factor $G(q)$ is temperature independent. Inclusion of the vertex corrections in the dielectric function through the local-field factor brings about considerable changes in the theory of metals. ${ }^{19}$ Figure 3 shows the BGR for a quantum wire of lateral width $a=500 \AA$, with the local-field corrections (dashed line) at $T=100 \mathrm{~K}$. We observe that within the simple Hubbard approximation to $G(q)$, the BGR deviates only slightly from the RPA result. The difference in $E_{p}$ with and without $G(q)$ is largely independent of temperature. We have also found good agreement for other values of the well width. These results suggest that the RPA is valid (in the range $10^{4}<N<10^{6} \mathrm{~cm}^{-1}$ ) provided that the local-field factor we use is correct. In order to assess a more reliable measure of corrections beyond the RPA, better approximations to the local field factor $G(q)$ are needed.

The temperature-dependent behavior of the mass renormalization is also a consequence of the dielectric function $\varepsilon(q, T)$, its main effect being to reduce the electron-phonon coupling. In the no-screening limit $[\varepsilon(q) \rightarrow 1]$, the effective mass (renormalized mass) is independent of temperature and carrier density

$$
\begin{aligned}
\frac{1}{m_{e, h}^{*}}= & \frac{1}{m_{e, h}}-\frac{\alpha_{e, h}}{m_{e, h}}\left[\frac{2}{\pi} \frac{\omega_{\mathrm{LO}}^{2}}{\sqrt{2 m_{e, h} \omega_{\mathrm{LO}}}} \frac{2}{m_{e, h}}\right. \\
& \left.\times \int_{0}^{\infty} d q F(q) \frac{q^{2}}{\left[\omega_{\mathrm{LO}}+q^{2} / 2 m_{e, h}\right]^{3}}\right],
\end{aligned}
$$

which we write as $1 / m^{*}=(1-\alpha B) / m$. In the opposite limit of infinite screening $(\varepsilon \rightarrow \infty)$ the electron (and hole) no longer couples to the phonon and there is no mass renormalization, i.e., $m_{e, h}^{*}=m_{e, h}$. Thus $m^{*}$ is bounded between the values $1 /(1-\alpha B)$ and 0.07 ( 0.4 for holes and in units of bare electron mass). In Figs. 5(a) and 5 (b) we display the percent change in the band masses for electrons and holes, respectively, as a function of temperature. To illustrate the density dependence, we show (by solid lines) from top to bottom $N=10^{6}, 10^{5}$, and $10^{4} \mathrm{~cm}^{-1}$ for a quantum wire of well width $a=200 \AA$. Indicated by the dotted lines are the no-screening limit results discussed above. We observe that mass renormalization is rather large for both electrons and holes, $\sim 6 \%$ and $10 \%$, respectively. We are not aware of any experiments to compare our results in quantum-well wires where the temperature dependence of the polaron mass is measured. In the case of two-dimensional (2D) systems, Das Sarma and Stopa ${ }^{13}$ found the mass renormalization to be rather small compared with the cyclotron resonance data. Since our analysis is along similar lines, we do not 

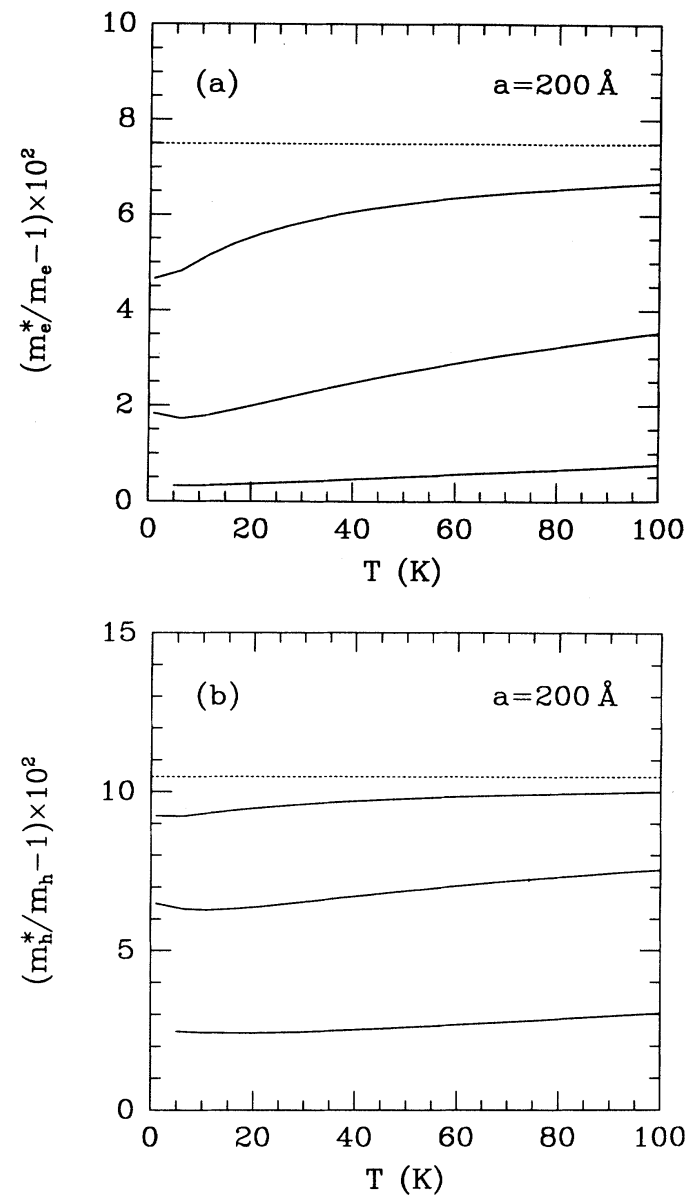

FIG. 5. (a) Effective mass renormalization at the conduction-band edge as a function of temperature for a quantum-well wire of width $a=100 \AA$. The solid lines from top to bottom indicate $N=10^{6}, 10^{5}$, and $10^{4} \mathrm{~cm}^{-1}$; the dotted line gives the no-screening limit. (b) Same for the valence-band edge.

expect to obtain good agreement with the cyclotron resonance experiments. It remains an open problem to develop an adequate theory of screening of electron-phonon coupling in high magnetic fields.

It has been noted ${ }^{13,20}$ that the static screening has a stronger effect in the renormalization than the dynamic screening, because in the static approximation only the long-time response of the system is taken into account. Similar conclusions are drawn by $\mathrm{Hai}$ et $a$ l. $^{14}$ in their calculation that takes the dynamic screening effects into account for quasi-one-dimensional systems. We have not attempted a perturbative calculation which includes dynamical screening because of the computational difficulties involved, but expect the polaronic corrections $E_{p}$ to increase in magnitude if such an approach is considered.

For the quasi-one-dimensional electron system we have used the model developed by $\mathrm{Hu}$ and Das Sarma, ${ }^{8}$ which introduces an additional confinement to an infinite square well. There are various other models of the quantumwell wire structures using parabolic confining potentials, and geometric reduction of dimensionality. The general trends obtained here for the plasma density and temperature dependence should be valid irrespective of the details of the model chosen.

\section{SUMMARY}

We have calculated the polaron self-energy in a quasione-dimensional GaAs quantum-well wire and found that its magnitude is comparable to the exchange-correlation effects. Our calculation is appropriate for a photoexcited semiconductor structure in which both electrons and holes take part in the screening. The coupling of the charge carriers to the LO phonons leads to a shrinkage of the band gap, which decreases as the plasma density is increased. We have found that a temperaturedependent plasmon-pole approximation to the dielectric function yields results qualitatively different from the RPA approximation, especially at high temperatures. Within our perturbative, on-shell approximation to the self-energies we have estimated the polaron effective mass and investigated its dependence on the temperature and carrier density. A simplified attempt is made to include the vertex corrections to the screening in the spirit of mean-field approximation. We find that the local-field corrections tend to not change the magnitude of the polaronic corrections significantly. Our analysis may be extended to doped $p$ - or $n$-type semiconductors in which either type of carrier is screened separately. Similarly, band gap renormalization due to the confined phonons would also be interesting.

\section{ACKNOWLEDGMENTS}

We gratefully acknowledge the partial support of this work by the Scientific and Technical Research Council of Turkey (TUBITAK) and fruitful discussions with Professor E. Kapon.
${ }^{1}$ H. Haug and S. Schmitt-Rink, Prog. Quantum Electron. 9, 3 (1984); S. Schmitt-Rink, D. S. Chemla, and D. A. B. Miller, Adv. Phys. 38, 89 (1989).

2 T. Demel, D. Heitmann, P. Grambow, and K. Ploog, Phys. Rev. B 38, 12732 (1988).
${ }^{3}$ J. Christen, M. Grundmann, E. Kapon, E. Colas, D. M. Hwang, and D. Bimberg, Appl. Phys. Lett. 61, 67 (1992).

${ }^{4}$ H. Haug and S. Schmitt-Rink, J. Opt. Soc. Am. B 2, 1135 (1985); S. Schmitt-Rink, C. Ell, S. W. Koch, H. E. Schmidt, and H. Haug, Solid State Commun. 52, 123 (1984); H. 
Haug and S. W. Koch, Phys. Rev. A 39, 1887 (1989).

${ }^{5}$ C. Ell, R. Blank, S. Benner, and H. Haug, J. Opt. Soc. Am. B 6, 2006 (1989); C. Ell, H. Haug, and S. W. Koch, Opt. Lett. 14, 356 (1989).

${ }^{6}$ G. Tränkle, Phys. Rev. B 36, 6712 (1987); Phys. Rev. Lett. 58, 419 (1987); G. Bongiovanni and J. L. Staehli, Phys. Rev. B 39, 8359 (1989); C. Weber, ibid. 38, 12748 (1988); V. D. Kulakovskii, ibid. 40, 8087 (1989).

${ }^{7}$ R. Cingolani, R. Rinaldi, M. Ferrara, G. C. La Rocca, H. Lage, D. Heitmann, K. Ploog, and H. Kalt, Phys. Rev. B 48, 14331 (1993); R. Cingolani, H. Lage, L. Tapfer, H. Kalt, D. Heitmann, and K. Ploog, Phys. Rev. Lett. 67, 891 (1991).

${ }^{8}$ B. Y.-K. Hu and S. Das Sarma, Phys. Rev. Lett. 68, 1750 (1992); Phys. Rev. B 48, 5469 (1993).

${ }^{9}$ S. Benner and H. Haug, Europhys. Lett. 16, 579 (1991).

${ }^{10}$ S. Das Sarma, R. Jalabert, and S.-R. Eric Yang, Phys. Rev. B 39, 5516 (1989); 41, 8288 (1990).

${ }^{11}$ L. Wendler, R. Haupt, and R. Pechstedt, Phys. Rev. B 43, 14669 (1991).
${ }^{12}$ Effects of interface phonons to the polaron energy in a quasi-two-dimensional electron gas have been considered in M. H. Degani and O. Hipólito, Phys. Rev. B 35, 7717 (1987).

13 S. Das Sarma and M. Stopa, Phys. Rev. B 36, 9595 (1987); W. Xiaoguang, F. M. Peeters, and J. T. Devreese, Phys. Status Solidi B 133, 229 (1986).

${ }^{14}$ G. Q. Hai, F. M. Peeters, J. T. Devreese, and L. Wendler, Phys. Rev. B 48, 12016 (1993).

${ }^{15}$ S. Das Sarma and B. A. Mason, Ann. Phys. (N.Y.) 163, 78 (1985).

${ }^{16}$ P. Maldague, Surf. Sci. 73, 296 (1978).

${ }^{17}$ Q. Li and S. Das Sarma, Phys. Rev. B 40, 5860 (1989); A. V. Chaplik and M. V. Krasheninnikov, Surf. Sci. 98, 533 (1980).

18 A. Gold and A. Ghazali, Phys. Rev. B 41, 7626 (1990).

${ }^{19}$ G. D. Mahan and B. E. Sernelius, Phys. Rev. Lett. 62, 2718 (1989).

${ }^{20}$ X. L. Lei, J. Phys. C 18, L731 (1985). 